\title{
Ámbitos de aplicación del Coaching educativo: una revisión bibliográfica del periodo 2013-17
}

\section{Applications of Educational Coaching: a literature review of research published during 2013-17}

\author{
Eva Ramos Loredo*1 \\ evar11025@gmail.com \\ Beatriz Sierra-Arizmendiarrieta** \\ bsierra@uniovi.es \\ Cristina Roces Montero** \\ croces@uniovi.es \\ *Colegio San Miguel, Gijón, España \\ **Universidad de Oviedo, España
}

\section{Resumen:}

El coaching es una metodología que persigue el desarrollo del máximo potencial personal. Recientemente, se ha trasladado al campo educativo para mejorar las habilidades y prácticas de los docentes y el alumnado. Los objetivos principales de esta investigación son: realizar una revisión bibliográfica de los últimos años, identificar los ámbitos de aplicación más recurrentes, y descubrir aquellos menos contrastados, de cara a sugerir nuevas líneas de investigación. La metodología está basada en una revisión sistemática de artículos científicos de los últimos cinco años en idioma español e inglés, distinguiendo los que son exclusivamente teóricos o los que dan cuenta de algún tipo de

\begin{abstract}
:
Coaching is a methodology whose purpose is to develop as much as possible the potential of individuals. It has been recently applied to the educational field in order to improve teaching skills and teacher and student performance. The aims of this research are: to carry out a review of current literature about coaching, to identify the most common application areas and to detect underdeveloped lines of research regarding coaching with a view to suggesting new approaches to this field. The methodology is based on a systematic review of articles, both in Spanish and English, published during the last five years. Besides, a distinction is made between theoretical and empirical studies.
\end{abstract}

1 Dirección para correspondencia (correspondence address):

Eva Ramos Loredo. Departamento sociolingüístico. Colegio San Miguel, Gijón. C/ Jovellanos, 2-12 A. 33201 Gijón, Asturias (España). 
Ámbitos de aplicación del Coaching educativo: una revisión bibliográfica del periodo 2013-17

Eva Ramos Loredo, Beatriz Sierra-Arizmendiarrieta Y Cristina Roces Montero

intervención. Como resultado, se constata una gran cantidad de estudios que, sobre el coaching educativo, se hacen hoy en día, así como su eficacia y beneficios para toda la comunidad educativa si tenemos en cuenta los artículos de carácter práctico. Se puede apreciar que en algunos países, como Estados Unidos, la presencia del coaching ya está muy arraigada, pero en realidad se trata de un fenómeno global que impregna en mayor o menor medida al sistema educativo de países de los cinco continentes. A pesar de ello, muchos autores consideran que es preciso incrementar el número de estudios empíricos sobre el coaching educativo y garantizar su rigor científico. Para finalizar, y teniendo en cuenta el corpus existente, se propone profundizar en una línea de investigación poco estudiada: el uso del coaching para mejorar los problemas de aprendizaje en secundaria.

\section{Palabras clave:}

Coaching; educación; revisión bibliográfica; función docente; innovación.
The current interest in educational coaching is reflected by the high number of studies published on this matter. Moreover, empirical studies confirm the effectiveness and benefits of coaching for all the agents involved in the educational community. Educational coaching has become a habitual practice in some countries such as The Unites States and is progressively becoming popular in educational settings all over the world. Despite this growing interest, multiple authors point out that more research is needed on educational coaching. They also consider that it is necessary to guarantee the scientific rigor of research. Finally, and taking into account the current corpus of studies, it seems necessary to explore a research line which to date has not been paid enough attention to: the use of coaching in secondary education when it comes to supporting adolescents with learning difficulties.

\section{Keywords:}

Coaching; education; literature review; teaching function; innovation.

\section{Résumé:}

Le coaching est une méthodologie qui poursuit le développement d'un potentiel personnel maximal. Il a récemment émergé dans le domaine de l'éducation pour améliorer les compétences et les pratiques des enseignants et des étudiants. Les principaux objectifs de cette recherche sont $d^{\prime}$ effectuer une revue bibliographique des dernières années, d'identifier les champs d'application les plus récurrents, et de découvrir ceux moins contrastés, afin de proposer de nouvelles lignes de recherche. La méthodologie est basée sur une revue systématique des articles scientifiques des cinq dernières années en espagnol et en anglais, en distinguant ceux qui sont exclusivement théoriques ou ceux qui présentent un type d'intervention. En conséquence, on vérifie qu'aujourd'hui il y a un grand nombre d'études sur le coaching éducatif, en constatant aussi leur efficacité et leurs avantages pour l'ensemble de la communauté éducative, si nous prenons en compte les articles pratiques. On peut remarquer que, bien que dans certains pays, comme aux États-Unis, la présence du coaching soit déjà profondément enracinée, mais il s'agit en réalité d'un phénomène global qui imprègne plus ou moins le système éducatif des pays des cinq continents. Malgré cela, de nombreux auteurs considèrent qu'il est nécessaire d'augmenter le nombre d'études empiriques sur le coaching éducatif et de garantir sa rigueur scientifique. Pour conclure, et sur la base du corpus existant, il est proposé de se plonger dans une ligne de recherche peu étudiée: I'utilisation du coaching pour améliorer les problèmes d'apprentissage à l'enseignement secondaire.

\section{Mots clés:}

Coaching; éducation; revue de la littérature; fonction d`enseignement; innovation.

Fecha de recepción: 17-7-2018

Fecha de aceptación: 23-9-2018 


\section{Introducción}

El coaching es un método, y un proceso, para aumentar la calidad de vida y el rendimiento de las personas, basado en el poder de la mente y de la palabra. Algunas fuentes de las que bebe son: la lingüística, la psicología -especialmente la psicología humanista y la psicología positiva-, y la programación neurolingüística. Su herramienta más eficaz es el diálogo entre el coach y el coachee, pero no es la única. Entre sus principales pilares se encuentran: la búsqueda del autoconocimiento, la concreción de metas y de un plan de acción para conseguirlas, el análisis reflexivo y la autoevaluación y la retroalimentación.

Su origen se remonta a la década de los años 70 del siglo pasado ligada al mundo del deporte, pero pronto saltó al campo empresarial donde ha cosechado grandes éxitos y se mantiene hoy día en rigurosa vigencia y actualidad. En función de los resultados obtenidos, el área de acción del coaching se expandió dando lugar a las distintas ramificaciones existentes: coaching educativo, coaching para padres, life coaching... En ocasiones se produce cierta confusión entre los términos coaching educativo y psicología positiva, aunque hay claros matices diferenciadores entre los que se puede destacar el carácter reactivo, el método y la planificación del coaching, frente al carácter proactivo, y la ausencia de método y planificación en la psicología positiva (Sandoval Lentisco y López Martínez, 2017).

El coaching educativo está pensado para ser empleado con el alumnado perteneciente tanto al sistema universitario como no universitario, con el fin de mejorar su bienestar y ayudarles a conseguir sus objetivos. Busca un cambio metodológico que conduzca a una forma distinta de ver el aprendizaje en la que no solo importen los resultados académicos, sino también el desarrollo madurativo de los estudiantes; donde el aprendizaje siga un modelo cooperativo desarrollando especialmente la competencia de aprender a aprender.

\section{Objetivos y método}

Los objetivos principales de este artículo son tres:

1. Realizar una revisión bibliográfica sobre el coaching educativo del periodo 2013-17. 
Ámbitos de aplicación del Coaching educativo: una revisión bibliográfica del periodo 2013-17

Eva Ramos Loredo, Beatriz Sierra-Arizmendiarrieta Y Cristina Roces Montero

2. Identificar los ámbitos de aplicación más recurrentes ligados al coaching educativo y clasificar los artículos en función de los mismos.

3. Comprobar si existen estudios sobre el coaching educativo orientados a ayudar a mejorar problemas de aprendizaje en alumnos de secundaria.

Esta es una revisión bibliográfica descriptiva, la cual permite al lector ponerse al día y actualizar la información que posee sobre cualquier área de conocimiento. Es especialmente útil en el campo de la educación, como en este caso, dadas las frecuentes propuestas innovadoras para mejorar el proceso de enseñanza-aprendizaje. El método de investigación ha pasado por cuatro fases: definición del problema, búsqueda de la información, organización de la información y análisis de la misma (Gómez-Luna, Fernando-Navas, Aponte-Mayor y Betancourt-Buitrago, 2014). Teniendo en cuenta dichas etapas, se ha procedido a realizar una revisión sistemática de artículos sobre el coaching educativo de los años 2013-17 en los idiomas español e inglés. En algún caso, por la relevancia del estudio, se ha incluido algún artículo del 2012.

La búsqueda se ha centrado mayoritariamente en artículos publicados en revistas científicas publicadas en papel y online, así como algunas aportaciones de interés encontradas en Actas de Congresos. Se han utilizado bases de datos nacionales e internacionales. En primer lugar, la estrategia de búsqueda se llevó a cabo a través de la base de datos WOS y SCOPUS de la Fecyt. Asimismo, se realizaron búsquedas complementarias a través de Google Scholar y de la base de datos bibliográfica del CSIC dedicada a las Ciencias sociales: ISOC. Los términos de búsqueda más utilizados fueron "coaching educativo", "educational coaching", "coaching school" y "coaching teenagers". También se utilizó como referencia el ranking de revistas españolas científicas de educación 2015 de la revista Comunicar, y se realizó una revisión de los índices de los números del periodo citado de revistas educativas JCR posicionadas en el primer y segundo cuartil.

En la búsqueda se seleccionaron un total de ciento tres estudios, quedando excluidos sesenta y dos que no se consideraron significativos para el objetivo de esta revisión por ser únicamente de índole divulgativa. La selección inicial se llevó a cabo por medio de la revisión de los abstracts o resúmenes y, después, a través de la lectura completa de los artículos 
con el fin de garantizar la relevancia del estudio para esta revisión. Finalmente, se escogieron cuarenta y un artículos, de los cuales 25 están en inglés, lo que supone casi el $61 \%$ del total y 16 están en español, lo que representa un 39\% aproximadamente.

\section{Análisis de la información y clasificación}

Para poder analizar la información, se han clasificado los artículos en dos grandes grupos: informes, revisiones o propuestas teóricas, por una parte, e investigaciones empíricas, resultados de algún tipo de intervención o implementación de programas diversos, por otra.

Tabla 1

Clasificación de artículos sobre el coaching educativo de los años 2013-17

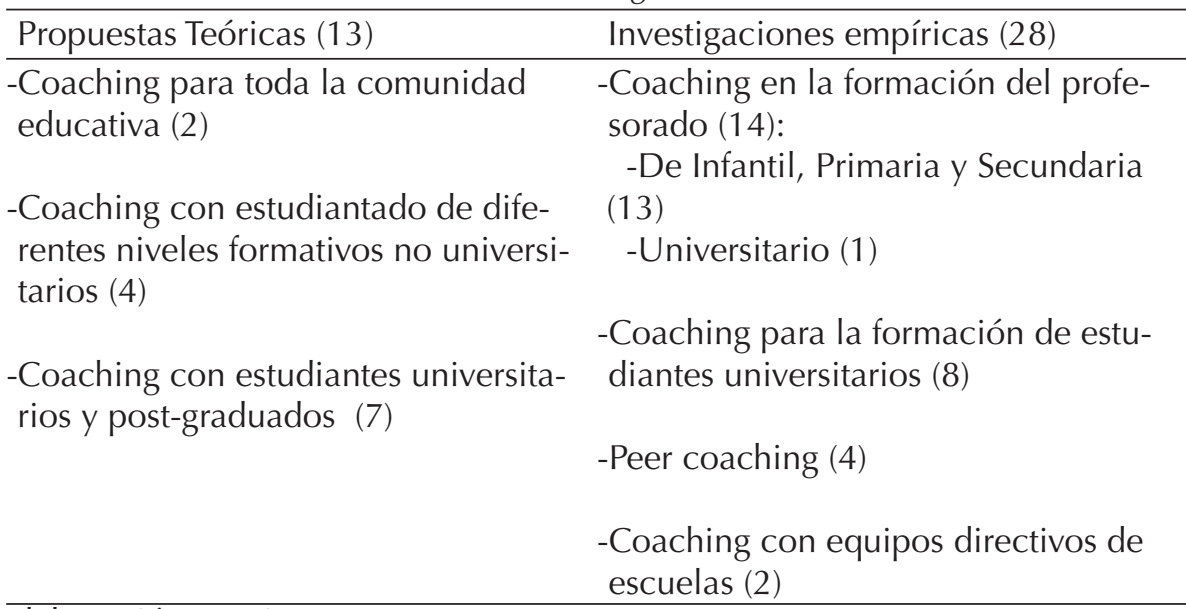

Elaboración propia

\section{Informes, revisiones o propuestas teóricas}

Al analizar los 13 artículos seleccionados, en esta modalidad, se han encontrado diferencias respecto a los destinatarios de la propuesta del uso del coaching. Hemos distinguido, en función de ello: a) beneficios de su uso para toda la comunidad educativa, destacando 2 artículos; b) beneficios para el estudiantado de diferentes niveles educativos no uni- 
Ámbitos de aplicación del Coaching educativo: una revisión bibliográfica del periodo 2013-17

Eva Ramos Loredo, Beatriz Sierra-Arizmendiarrieta Y Cristina Roces Montero

versitarios, centrándonos en 4 artículos; c) beneficios del coaching para estudiantes universitarios y postgraduados, con 7 estudios.

\section{Coaching para toda la comunidad educativa}

Salavert (2015) señala los beneficios del coaching en la enseñanza: su impronta sobre la capacidad de liderazgo, tanto en el equipo directivo como en los profesores, la mejora general de la calidad docente y el aumento de rendimiento en el estudiantado aunado con una mejor preparación para afrontar los retos que les depare su futuro. Indica que el uso del coaching mejora las relaciones con los compañeros de trabajo y la motivación de los docentes para continuar perfeccionando sus prácticas hasta lograr el éxito educativo para todos sus estudiantes. La repercusión de este cambio en los procesos de enseñanza-aprendizaje se traduce en que facilita la creación de un ambiente en el que el alumnado se siente apoyado. Potencia, además, la adquisición de conocimiento y de pensamiento crítico.

Asimismo, Bécart y Ramírez (2016) establecen una relación entre el uso del coaching y el desarrollo de las competencias categorizadas en saber ser-saber-convivir, saber-hacer y saber-aprender.

\section{Coaching con estudiantado de diferentes niveles formativos no universitarios}

Evans (2013) y Hernández-Aristu y Hernández-Van-Waes (2016) sugieren el uso del coaching para solucionar las principales dificultades a las que deben enfrentarse los adolescentes. El primero propone el uso de grupos de discusión, talleres, coaching... para facilitar su proceso de autorregulación. Los segundos, ponen el foco de atención en su falta de responsabilidad, autonomía y consciencia en general y argumentan que la tutoría, la orientación y el coaching individual pueden dar respuesta a esas carencias.

Davies et al. (2013) ponen de manifiesto la importancia de la creación de un entorno creativo en la educación para mejorar los resultados académicos de los estudiantes y su desarrollo personal. Proponen, así, el coaching para mejorar la creatividad del alumnado.

Pocos estudios vinculan especialmente el uso de técnicas de coaching con los adolescentes. Entre ellos, Sánchez-Teruel (2013) considera 
que podría llevarse a cabo de manera sencilla, contando con la colaboración de un coach externo o mediante la formación del profesorado para crear la figura del docente-coach. Señala que esta metodología podría ser muy beneficiosa para docentes y alumnado. Para los primeros, por el incremento de confianza entre profesor y alumno, y la mejora en la predisposición al cambio. Para los estudiantes, por el desarrollo de las habilidades propias, la motivación y técnicas para aprender a aprender.

\section{Coaching con estudiantes universitarios y post-graduados}

Karpińska-Musiał y Dziedziczak-Foltyn (2014) apuestan por la introducción de técnicas como el tutoring y el coaching en la educación superior polaca para mejorar los procesos de enseñanza-aprendizaje. Estos autores aseguran que ambos métodos interconectados pueden devolver a la educación superior polaca al más alto nivel. Sobre el coaching afirman que es capaz de desarrollar la capacidad cognitiva del estudiantado al máximo, gracias a algunas de sus estrategias: tomar conciencia, reflexionar, marcarse metas, analizarse objetivamente incluyendo aptitudes y debilidades... Sin embargo, advierten de que su efectividad dependerá de la implicación y actitud del alumnado.

Bécart (2016) recomienda el uso del coaching, en la educación superior, por su capacidad para desarrollar competencias tales como la iniciativa personal, la inteligencia emocional y por su contribución a la hora de desarrollar de manera holística a las personas y de ayudarlas a definir sus objetivos. Asimismo, aconseja su integración en los currículos oficiales.

Baquero y Rodríguez-Moneo (2016) defienden el uso del coaching como una herramienta para desarrollar la capacidad de autorregulación, esencial en competencias tales como la de aprender a aprender, recomendando realizar estudios especialmente con estudiantes de educación superior por su carga superior de trabajo.

Fernández-Salinero (2014) y Castaño, Blanco y Asensio (2012) proponen el uso del coaching en las tutorías universitarias con el fin de lograr el desarrollo completo del estudiante y su bienestar, así como lograr su desarrollo como sujeto autónomo.

Respecto a los estudiantes de postgrado, Van del Weijden, Teelken, de Boer y Drost (2016) afirman que las universidades deberían realizar sesiones de coaching con los estudiantes que acaban de finalizar su 
doctorado, puesto que una vez terminados sus estudios se suelen sentir perdidos, ya que no saben hacia dónde reconducir su carrera fuera del ámbito académico. Por otro, Van Lankveld et al. (2017) recomiendan el uso del coaching en la formación de los médicos residentes para ayudarlos a identificarse con el nuevo rol que deben representar como profesores de una nueva generación de futuros médicos.

\section{Investigaciones empíricas}

Jiménez (2012) realiza una revisión hasta el año 2012 de estudios empíricos sobre el coaching como estrategia utilizada en la formación del profesorado. En este apartado se prosigue dicha revisión, pero se clasifican desde otra perspectiva, ampliando los destinatarios. Así, los artículos seleccionados se han clasificado en función de: a) la formación del profesorado, con 12 artículos en niveles preuniversitarios, y solo 1 en el campo universitario; b) la formación de estudiantes universitarios, con 9 artículos; c) peer-coaching o entre pares, con 4 experiencias; d) la formación de equipos directivos, con 2 estudios.

\section{Coaching en la formación de profesorado}

Siguiendo el mismo criterio que en el apartado anterior, se distinguirá entre la formación del profesorado de educación infantil, primaria y secundaria y la del universitario, reflejando la práctica del coaching en diversos países.

- Formación del profesorado de Educación Infantil, Primaria y Secundaria:

El objetivo de aplicar el coaching a la formación del profesorado es el de mejorar sus prácticas educativas en el aula. Vernon-Feagans, Kainz, Hedrick Ginsberg y Amendum (2013) demostraron la eficacia de este tipo de programas para mejorar las competencias de los maestros de educación infantil y primaria para enseñar a leer a su alumnado. El programa de coaching por ellos propuesto, fue realizado a través de una webcam con docentes de escuelas rurales de varios estados de Estados Unidos. A través de la webcam, los coaches iban enseñando a los maestros nuevas estrategias y herramientas para la enseñanza de la lectura. 
Los alumnos con dificultades pertenecientes al grupo experimental superaron significativamente a los alumnos con dificultades pertenecientes al grupo de control. Los autores confirmaron que el uso de tales métodos puede prevenir fracasos lectores en cursos posteriores.

En la misma línea, Early, Maxwell, Ponder y Pan (2017) analizan los resultados de dos intervenciones con alumnado de preescolar. En una de ellas un coach debía orientar, guiar y ayudar a reflexionar al profesor sobre su relación con sus discentes para mejorar la interacción y comunicación con ellos. El apoyo emocional de los profesores aumentó en comparación con el grupo de control y valoraban más sus actividades de desarrollo profesional. También Slot, Leseman, Verhagen y Mulder (2015) utilizaron el coaching en la formación del profesorado de educación infantil.

El objetivo del uso del coaching en Primaria para Matsumura, Garnier y Spybrook (2012) es promover los debates en el aula a partir de los textos de lectura y así favorecer la alfabetización. Como innovación, proponen contrastar la eficacia del programa habitual en Estados Unidos de "literacy coaching" y otro más evolucionado: el "content-focused coaching". Tras dos años de puesta en marcha, el programa demostró tener un efecto positivo y significativo en la calidad de las reflexiones sobre los textos, lo cual vendría a demostrar que un programa de coaching bien diseñado centrado en la práctica del debate puede mejorar la calidad de los debates en el aula.

El estudio de Reinke, Stormont, Webster-Stratton, Newcomer y Herman (2012) se presenta como un ejemplo de cómo el coaching se ha venido usando como un sistema de apoyo en el programa de control de la clase "the Incredible Years Teacher Classroom Management" en Estados Unidos. En él, el coaching actúa apoyando a los profesores y ayudándoles a llevar a la práctica nuevas técnicas y habilidades que han descubierto a lo largo del programa y ayudándoles a averiguar en qué contextos su uso resulta más apropiado. Los docentes, con alumnado de 3 a 8 años, recibieron talleres y sesiones de coaching individuales. Entre los beneficios del apoyo del coach destacan: la retroalimentación, estrategias efectivas para tratar con los niños, ayuda para seleccionar sus objetivos, y generar confianza en sí mismos al destacar sus triunfos. También Akiba y Wilkinson (2016) recomiendan el empleo de coaches externos en las escuelas para elevar el nivel académico de los centros educativos, a través del trabajo con los docentes ayudándoles a desarroIlar auténticas lesson studies. 
Becker, Bradshaw, Domitrovich y lalongo (2013) demuestran que el uso del coaching suponía una contribución muy valiosa a la implementación de programas de prevención en el aula. Para ello, contaron con la participación de 129 maestros de educación primaria de distintas zonas urbanas de Estados Unidos.

Kunz, White, Smith, y Nugent (2014) distinguen entre aquellas actuaciones y estrategias propias del coaching que resultan realmente efectivas en el ámbito educativo y aquellas prácticas que no lo son. Así, Ilamaron "ingredientes activos" a las actividades relevantes, entre las que se encuentran: el uso del rapport, el feedback, la actitud comprometida del profesor con el proyecto, la discusión reflexiva y la elaboración de un plan de acción.

En EE.UU. el coaching está más desarrollado, pero también podemos encontrar investigaciones con profesorado de primaria en otros lugares. En Puerto Rico, se han realizado estudios similares. Colorado y Corcino (2014) reflejaron la opinión de una serie de maestros de primaria acerca del coaching tras haber participado en sesiones de formación. Más del 90\% de los maestros calificó la práctica como excelente o muy buena y la recomendarían a otros colegas de profesión.

Piper y Zuilkowski (2015) evaluaron los resultados de un programa similar en escuelas de África. Sus datos proceden de ocho mil estudiantes de un colegio de Primaria de Kenia participante en una prueba estatal sobre lectura y matemáticas. Los resultados reflejaron que a más visitas por parte de los coaches, mejor rendimiento obtenían los alumnos, y que la disminución del número de profesores dirigidos por el mismo coach, aumenta los resultados del alumnado ya que puede prestarles una atención más personalizada, especialmente en estudios no reglados.

Twigg, Pendergast, Fluckiger, Garvis, Johnson, y Robertson, J. (2013) evaluaron un programa de coaching desarrollado por el Departamento de Educación y Desarrollo de la Primera Infancia de Australia. La evaluación del programa demostró que el coaching resultó efectivo para ayudar a los docentes a alcanzar los objetivos del programa marco, además de facilitar la reflexión y dotar a los participantes del apoyo necesario para animarlos a asumir y adoptar los cambios. De la misma manera, en Gran Bretaña, Perryman, Ball, Braun y Maguire (2017) demostraron que el juicio externo necesario para el proceso de coaching es en muchas ocasiones recibido muy positivamente, al ser interpretado como un camino para la superación y mejora de uno mismo. 
Ya en centros de educación secundaria, Day, Gu y Sammons (2016) describen un programa de intervención de 4 fases llevado a cabo en Eyhampton High School, en Reino Unido. Este centro, ubicado en un barrio desfavorecido, se caracterizaba por el mal comportamiento y rendimiento de sus estudiantes. Un nuevo director puso en marcha el citado programa en cuya fase dos incluía el uso del coaching con el profesorado para mejorar sus estrategias a través de la observación y señalación de sus debilidades y fortalezas, lo que se tradujo en una mejora en la actitud y rendimiento del alumnado.

- Formación del profesorado universitario:

Maldonado, Sánchez y Mendoza (2014) han desarrollado un programa basado en el coaching para mejorar la docencia universitaria. Para ello, contaron con la participación de ocho profesores de la Universidad Pedagógica Experimental Libertador, en Venezuela y de un facilitadorcoach para guiarlos. Algunas de las estrategias empleadas por el coach fueron: la observación, la escucha activa y la formulación de preguntas. Finalizado el programa, todos los agentes del mismo reflejaron que los profesores lograron desarrollar o reforzar una serie de habilidades esenciales para el trato con el alumnado y para un hipotético futuro trabajo de colaboración con otros colegas. Además, obtuvieron conocimiento de lo que antes carecían y fueron capaces de elaborar su propio plan de acción para conseguir sus objetivos.

\section{Coaching para la formación de estudiantes universitarios}

Otra área especialmente trabajada desde el coaching educativo en los últimos tiempos es el impacto en la formación de estudiantes universitarios. Bettinger y Baker (2013) realizaron una investigación con estudiantes universitarios de Estados Unidos en el que probaron la eficacia del uso del método coaching individualizado para evitar el abandono de los estudios universitarios. Para ello, se sirvieron de los datos aportados por el programa "Inside Track", que ofrece como servicio la práctica de coaching con el estudiantado de universidades públicas y privadas. Los resultados fueron muy satisfactorios: los estudiantes del grupo experimental, en líneas generales, presentaron una mayor asistencia a la universidad hasta un año después de finalizado el programa.

En México, Arzate (2013) aplicó un programa educativo basado en el 
Ámbitos de aplicación del Coaching educativo: una revisión bibliográfica del periodo 2013-17

Eva Ramos Loredo, Beatriz Sierra-Arizmendiarrieta Y Cristina Roces Montero

coaching en dos grupos de la Licenciatura de Educación Primaria de la Escuela Normal para profesores (CyBENP). Ambos grupos aumentaron su motivación y desarrollaron aprendizajes significativos, mejoraron las relaciones sociales en el aula, desarrollaron un plan de vida y se responsabilizaron y tomaron conciencia de su futuro, aumentaron su autocontrol y su impulsividad y mejoraron en el uso de las TIC y en la búsqueda de información; aunque solo uno de los grupos alcanzó el desempeño académico esperado.

También en México, Rodríguez y Martínez (2015) querían constatar la eficacia de un programa de coaching, implementado a lo largo de cuatro meses, con estudiantes de tercer curso de ingeniería farmacéutica. Este programa estaba especialmente enfocado a favorecer el desarrollo de las capacidades de autorregulación del aprendizaje en los discentes. Los resultados obtenidos demuestran la eficacia del proceso y destacan el desarrollo del uso de estrategias de aprendizaje y el manejo del ambiente y la conducta. Además, añaden el aumento de la responsabilidad, el compromiso, la autoeficacia, la autoconfianza e incluso la calidad de las tareas entregadas.

En Indonesia Purwa Udiutoma, Srinovita y Si (2015) pusieron en marcha su proyecto a partir de las conocidas como becas Beastudi Etos para estudiantes universitarios, en las que proveían a los beneficiarios no solo de financiación, sino también de sesiones de coaching y mentoring. Entre las habilidades desarrolladas destacan: la capacidad de liderazgo, el trabajo en equipo, la motivación y la habilidad para comunicarse, así como otras de tipo actitudinal como la mejora en las relaciones interpersonales.

En España, destaca la investigación de Rosa, Riberas, Navarro-Segura y Vilar (2015), que refleja los beneficios de la introducción del coaching en diversas asignaturas del grado de Educación Social y Trabajo Social de la universidad Ramón Llul, con la finalidad de desarrollar la competencia emocional en su alumnado. Entre sus resultados, señalan que favorece que el estudiantado manifieste sus sentimientos y pensamientos respetuosamente. También mejora su capacidad de escucha y de búsqueda de alternativas, al mismo tiempo que les ayuda a aceptar sus errores y a transformarlos en oportunidades para aprender y seguir creciendo.

Los tres siguientes proyectos coinciden en implementar un programa de coaching con estudiantes universitarios de disciplinas relacionadas 
con la formación de docentes. Rodríguez-Hidalgo, Calmaestra y Maestre (2015) optan por un nuevo modelo de Practicum cuya metodología se basa en el Aprendizaje Basado en Problemas y el coaching multidimensional. Estos autores han probado su eficacia para el desarrollo de las competencias en los estudiantes de Grado de Educación Primaria. Los resultados obtenidos han sido muy positivos, especialmente en competencias transversales tales como: reconocer la diversidad y la multiculturalidad, aprender de forma autónoma, adaptarse a nuevas situaciones, comprometerse éticamente, razonar de forma crítica y tomar la iniciativa con espíritu emprendedor. Y, asimismo, el desarrollo de competencias específicas de los maestros, como: promover la mejora de las relaciones interpersonales y las habilidades comunicativas, el trabajo en equipo, la toma de decisiones...

Sánchez y Boronat (2014) buscaron, esencialmente, el desarrollo de competencias intra e interpersonales que les ayuden a convertirse en profesionales de gran valor. Su estudio reflejó la percepción de los propios alumnos sobre su aprendizaje: un $65 \%$ percibió un aumento en su autoestima, un $78 \%$ un aumento en su motivación, más del $50 \%$ se considera más eficaz a la hora de resolver problemas y tomar decisiones individualmente y de forma conjunta con sus compañeros.

Finalmente, Maestre, Nail y Rodríguez-Hidalgo (2017) implementaron un programa basado en el Aprendizaje Basado en problemas y en el coaching para mejorar los niveles de competencia de las TICs y de educación inclusiva con profesorado de educación primaria durante el Prácticum. El estudio demostró una mejoría significativa en las competencias de educación inclusiva: resolución de conflictos, reconocimiento y respeto por la diversidad, convivencia, gestión de relaciones interpersonales. Pero de las competencias relacionadas con las TICs la única en la que se percibe una mejora es la relacionada con aspectos comunicativos.

\section{Peer coaching}

El Peer coaching supone el empleo de técnicas de coaching, tales como la observación y la retroalimentación entre iguales, ya sean dos estudiantes o dos profesores. Los beneficios que reporta han sido estudiados por Dulagil, Green y Ahern (2016), quienes publicaron un estudio basado en la implementación de un programa de coaching, dentro de un programa 
de educación positiva en un instituto australiano y con una muestra de 25 estudiantes adolescentes, todas mujeres, entre 15 y 16 años de edad. El resultado del programa fue positivo, especialmente en las variables relacionadas con el aumento de los niveles de bienestar, así como un aumento significativo de la resiliencia. Además, las estudiantes manifestaron sentir que tenían más éxito en sus metas personales, pero no notaron diferencia en las académicas.

Yee (2016) defiende el uso del coaching entre el profesorado de los centros educativos como forma de aprender nuevas estrategias pedagógicas, reflexionar sobre las posibilidades de mejora y las técnicas que no funcionan a través de la observación y retroalimentación. La confianza y honestidad entre los docentes es esencial para lograr su desarrollo profesional y, en consecuencia, mejorar el aprendizaje del alumnado.

De Rijdt, van der Rijt, Dochy y van der Vleuten (2012) compararon el desempeño de la tarea de tutor universitario con estudiantes de primer curso, llevado a cabo por parte del profesorado y por el alumnado senior. Los discentes habían sido formados a través de un curso intensivo para desarrollar sus habilidades de tutor que incluía peer coaching y a lo largo del año siguiente continuaron asistiendo a sesiones de coaching personal. Los resultados demostraron que no había habido diferencia en la percepción del desempeño de la función del tutor entre el profesorado y el alumnado.

Asimismo, Hoffman y Duffy (2017) han diseñado un programa para mejorar la eficacia de los profesores de alfabetización a partir del coaching, Ilamado coaching with care. Lo llevan a cabo entre dos profesores: uno en prácticas y otro experto y utilizan las técnicas del coaching para lograr la reflexión acerca de las prácticas docentes, la capacidad de adaptación a las situaciones reales que se dan en el aula y a pensar de manera diferente a lo usual para concebir todas las alternativas posibles.

\section{Coaching con equipos directivos de escuelas}

Hemos visto cómo una serie de estudios demostraban la eficacia del empleo del coaching con docentes de todos los niveles educativos para mejorar las prácticas del aula, así como en la formación de estudiantes universitarios y entre iguales. No obstante, esta no es la única utilidad del coaching para las escuelas, también puede servir y sirve como revulsivo para realizar cambios a nivel global, influyendo especialmente en el 
equipo directivo. Esta función del coaching ha sido estudiada por Mayer, Woulfin y Warhol (2015), y Kraft y Gilmour (2016). Los primeros se basaron en la "Together Initiative", una propuesta del gobierno de Estados Unidos para mejorar la calidad educativa en las escuelas. Este programa se puso en práctica en 8 escuelas de zonas urbanas de estados del norte del país y para su desarrollo contó con la colaboración de cuatro coaches. Este estudio reflejó que en un primer momento, los docentes atribuían a los coaches el papel de líderes de la reforma, aunque realmente eran aquellos los que realizaban todos los cambios importantes. La figura del coach resultó clave para que los maestros se percatasen de su capacidad para emprender dichas reformas.

También Kraft y Gilmour (2016) recogen las opiniones de una gran mayoría de directores de colegios de infantil, primaria y secundaria de un distrito del noreste de Estados Unidos que demandaban más formación para ellos, especialmente en cuestiones de coaching, con el fin de desarrollar habilidades de observación y retroalimentación con las que poder contribuir a la mejora del desarrollo profesional de los docentes viendo, además, la necesidad de contratar coaches externos.

\section{Conclusión y prospectiva}

Desde la década de los años 70, el coaching ha recorrido un largo camino, extendiéndose a varias áreas. Entre ellas, cabe destacar la educación, ya que algunos de los elementos más importantes del coaching como motivación, autoconocimiento, reflexión, planteamiento de objetivos, evaluación... son herramientas clave para el triunfo educativo. El coaching educativo ha crecido mucho en los últimos años y se ha multiplicado el número de artículos que abordan el tema.

Una de las definiciones más citadas entiende el coaching como la liberación del "potencial de una persona para incrementar al máximo su desempeño. Consiste en ayudarle a aprender, en lugar de enseñarle" (Whitmore, 1992, 10). De forma muy resumida, ello implica que el desarrollo y pleno rendimiento personal y profesional ha de partir del propio potencial y de las decisiones que llegue a tomar para desplegarlo y hacerlo rendir al máximo. El coaching es un método para ayudar a la reflexión, establecimiento de objetivos, toma de decisiones y diseño de estrategias para alcanzar lo deseado. Por eso, el coach no aporta conoci- 
Ámbitos de aplicación del Coaching educativo: una revisión bibliográfica del periodo 2013-17

Eva Ramos Loredo, Beatriz Sierra-Arizmendiarrieta Y Cristina Roces Montero

miento, sino que apoya y ayuda a desarrollar la confianza y motivación necesarias para recorrer el arduo camino del desenvolvimiento personal. Al hablar de coaching educativo, podría pensarse que el destinatario es básicamente el alumnado de las diferentes etapas educativas. Pero a lo largo de estas páginas y de la revisión llevada a cabo hemos podido comprobar que los destinatarios son varios: docentes, estudiantes de diferentes niveles, equipos directivos de centros educativos... Este método está progresivamente creciendo y desarrollándose. Y, además, su expansión difiere mucho en función del país que se tenga en cuenta. Hemos observado que hay estudios sobre coaching educativo en los cinco continentes, aunque no en todos, ni en todos los países de cada uno de ellos su implantación y práctica está igualmente extendida. En Estados Unidos, el uso del coaching en las escuelas es una práctica habitual y además su implantación está financiada por la administración pública; mientras que en España se está dando a conocer tímidamente.

Hasta hace unos años, los únicos estudios sobre el coaching educativo tenían un carácter meramente divulgativo y no buscaban contrastar la eficacia del método. Esta puede ser una de las posibles razones por las que todavía hay organismos reticentes a la incorporación del coaching en los centros educativos. Afortunadamente, esta situación ha ido evolucionando. Ya podemos encontrar estudios, con datos contrastados, que proporcionan información más útil para el futuro del coaching, de los cuales hemos pretendido dar cuenta en el presente artículo.

Nuestro primer objetivo se ha logrado, por tanto, con la revisión bibliográfica mostrada del periodo 2013-17 con la que se ha tratado de dar una visión lo más global posible de los avances en este campo. Los artículos se han distribuido en dos grandes apartados, dependiendo de que fueran propuestas teóricas o investigaciones empíricas, clasificándose ambos en función de los destinatarios del coaching, como se ha indicado al inicio de este apartado. Sin entrar a resumir nuevamente las principales aportaciones de las investigaciones referidas, es importante señalar la conexión de los beneficios del coaching con el desarrollo de habilidades y competencias de carácter trasversal. En las investigaciones realizadas con estudiantes se concluye que no se aprecia una mejora significativa en contenidos, pero sí un claro desarrollo en aspectos relacionales y comunicativos, autoconfianza, mayor iniciativa y reflexión, entre otros. Esto parece evidenciar que el coaching dirigido a estudiantes facilita el desarrollo de la competencia comunicativa, aprender a aprender, 
autonomía, competencia emocional... Por su parte, el coaching como método para la formación del profesorado resulta de ayuda sobre todo para mejorar la comunicación con el alumnado y desarrollar nuevas técnicas y métodos. A la vista de los resultados, podría decirse que sería una inestimable herramienta para evitar o paliar, al menos, la resistencia al cambio haciendo al profesorado consciente de sus fortalezas y capaz de afrontar cualquier tipo de reforma.

El segundo objetivo giraba en torno a la identificación de los ámbitos de aplicación más recurrentes del coaching educativo y su clasificación. Una vez analizada la información se puede concluir que una de las áreas más fructíferas es la formación del profesorado. La revisión de Jiménez (2012) realizada hasta el 2012, y centrada en la formación del profesorado, respalda y coincide con el mayor número de artículos encontrados sobre este tema en el siguiente quinquenio. De la totalidad de artículos referenciados, los dedicados a la formación del profesorado suponen cerca de un $35 \%$ y, teniendo en cuenta sólo las investigaciones empíricas, la formación docente ocupa un 50\% de los estudios. En segundo lugar, después de la formación docente, las investigaciones encontradas reflejan un mayor interés y utilidad en el coaching con estudiantes universitarios, con cerca de un $37 \%$ en relación con la totalidad de artículos y un $28 \%$ de las investigaciones de carácter empírico. Sorprende que el interés por la aplicación de programas de coaching se centre en el estudiantado del ámbito académico, no encontrando investigaciones, salvo propuestas teóricas, para alumnado de otros niveles educativos. Sin embargo, con el profesorado ocurre a la inversa, refiriéndose casi la totalidad de los estudios a la formación del profesorado de educación infantil, primaria y secundaria, sin apenas incidencia en el profesorado universitario.

Con respecto al tercer objetivo planteado, y en línea con lo mencionado en el punto anterior, no se han encontrado estudios empíricos sobre la aplicación del coaching a la mejora de los problemas de aprendizaje del alumnado de educación secundaria. Esto refuerza la necesidad e importancia de profundizar en esta línea de investigación, que ha de ir vinculada -desde nuestro punto de vista- a la formación docente, facilitando al profesorado herramientas y conocimientos propios de esta disciplina que puedan aplicar en sus clases, y analizando y contrastando las mejoras producidas en diferentes materias. Todos los estudios dirigidos a la formación docente que se han reseñado utilizan el coaching 
Ámbitos de aplicación del Coaching educativo: una revisión bibliográfica del periodo 2013-17

Eva Ramos Loredo, Beatriz Sierra-Arizmendiarrieta Y Cristina Roces Montero

como medio de apoyo al profesorado para el logro de diferentes objetivos, pero se echa de menos investigaciones empíricas que consideren el coaching como un contenido de formación más, de forma que puedan ejercer lo que Sánchez-Teruel (2013) denomina el docente-coach. Pensamos que si el coaching se incluyera de algún modo como contenido formativo, sea en cursos de formación continua o incluso en la formación inicial, sería interesante añadir esta faceta como una nueva función o rol del profesorado. Ello facilitaría a los docentes saber crear un clima de confianza que, junto con escuchar y preguntar -tareas básicas del coach- (Giráldez Hayes y van Nieuwerburgh, 2016, 32), pudiera ayudar al alumnado a superar debilidades derivadas de la falta de confianza, de autonomía, de iniciativa y de reflexión que ralentizan e incluso impiden su aprendizaje.

\section{Referencias bibliográficas}

Akiba, M. y Wilkinson, B. (2016). Adopting an International Innovation for Teacher Professional Development: State and District Approaches to Lesson Study in Florida. Journal of Teacher Education, 67(1), pp. 74-93. https://doi. org/10.1177/0022487115593603

Arzate, O. (2013). Coaching educativo: una propuesta metodológica para innovar en el aula. Ra Ximh Un estudio fenomenológico: posicionamiento y gestos en clase

Emmanuelle Maitre De Pembroke y traducido por Ana Castelo Garrido Un estudio fenomenológico: posicionamiento y gestos en clase

Emmanuelle Maitre De Pembroke y traducido por Ana Castelo Garrido ai, 9 (4), pp. 177 185. file:///C:/Users/Profesor/Downloads/54035-153674-1-PB\%20(1).pdf

Becker, K. D., Bradshaw, C. P., Domitrovich, C. y lalongo, N. S. (2013). Coaching teachers to improve implementation of the good behavior game. Administration and Policy in Mental Health and Mental Health Services Research, 40 (6), pp. 482-493. DOI: 10.1007/s10488-013-0482-8.

Baquero, J. A. y Rodríguez-Moneo, M. (2016). La relación entre el proceso de autorregulación y el proceso de coaching. Universitas Psychologica, 15, (1), pp. 15-25. DOI:10.11144/Javeriana.upsy15-1.rpap. http://www.scielo.org.co/pdf/rups/v15n1/ v15n1a11.pdf

Bécart, A. (2016). Coaching y fomento del emprendimiento: nuevas perspectivas para la educación superior. Perspectiva socioeconómica, 1 (3), pp. 17-27.

Bécart, A. y Ramírez Garrido, J.D. (2016). Fundamentos del coaching educativo: caracterización, aplicaciones y beneficios desde los cuatro pilares del saber. Plumilla educativa, 18, pp. 344-361. https://www.researchgate.net/publication/315739981/ download

Bettinger, E. P. y Baker, R. B. (2014). The Effects of Student Coaching An Evaluation of 
Ámbitos de aplicación del Coaching educativo: una revisión bibliográfica del periodo

Eva Ramos Loredo, Beatriz Sierra-Arizmendiarrieta Y Cristina Roces Montero

a Randomized Experiment in Student Advising. Educational Evaluation and Policy Analysis, 36 (1), pp. 3-19. DOI: 10.3102/0162373713500523.

Castaño Perea, E., Blanco Fernández, B. y Asensio Castañeda, E. (2012). Competencias para la tutoría: una experiencia de formación con profesores universitarios. Revista de Docencia Universitaria, 10 (2), pp. 193-210. file:///C:/Users/Profesor/Downloads/ Dialnet-CompetenciasParaLaTutoria-4021056.pdf

Colorado Laguna, R.J. y Corcino Marrero, L. (2014). Percepción y receptividad al proceso de coaching como componente de un programa de desarrollo profesional para maestros de escuela primaria. Paradigma, 35 (1), pp. 79-102.

Day, C., Gu, Q. y Sammons, P. (2016). The impact of leadership on student outcomes: how successful school leaders use transformational and instructional strategies to make a difference. Educational Administration Quarterly, 52 (2), pp. 221-258. https:// pdfs.semanticscholar.org/01a7/8b3b2e4000b7c68403af78eff914339baaf4.pdf

Davies, D., Jindal-Snape, D., Collier, C., Digby, R. Hay, P. y Howe, A. (2013). Creative learning environments in education- a systematic literature review. Thinking skills and Creativity, 8, pp. 80-91. https://www.sciencedirect.com/science/article/pii/ S187118711200051X

De Rijdt, C. C. E., van der Rijt, J. W., Dochy, F. y van der Vleuten, C.P.M. (2012). Rigorously selected and well trained senior student tutors in problem based learning: Student perceptions and study achievements. Instructional Science, 40 (2), pp.397-411. https://link.springer.com/article/10.1007/s11251-011-9173-6

Dulagil, A., Green, S., Ahern, M. (2016). Evidence-based coaching to enhance senior students' wellbeing and academic striving. International Journal of Wellbeing, 6 (3), pp. 131-149. doi:10.5502/ijw.v6i3.426 https://internationaljournalofwellbeing.org/index. php/ijow/article/viewFile/426/575

Early, D.M., Maxwell, K.L., Ponder, B.D. y Pan, Y. (2017). Improving teacher-child interactions: A randomized controlled trial of Making the Most of Classroom Interactions and My Teaching Partner professional development models. Early Childhood Research Quarterly, 38, pp. 57-70. http://dx.doi.org/10.1016/j.ecresq.2016.08.005 http://daneshyari.com/article/preview/4938242.pdf

Evans, C. (2013). Making sense os assessment feedback in higher education. Review of Educational Research, 83 (1), pp. 70-120. DOI: 10.3102/0034654312474350 http:// journals.sagepub.com/doi/abs/10.3102/0034654312474350

Fernández-Salinero Miguel, C. (2014). La tutoría universitaria en el escenario del espacio europeo de educación superior: perfiles actuales. Teoría educativa, 26 (1), pp. 161-186.

Gibbons, L.K. y Cobb, P. (2017). Focusing on Teacher Learning Opportunities to Identify Potentially Productive Coaching Activities. Journal of Teacher Education, 68 (4), pp. 411-425. https://doi.org/10.1177/0022487117702579

Giráldez Hayes, A. y van Nieuwerburgh, C. (2016). Coaching educativo. Madrid: Paraninfo.

Gómez-Luna, E., Fernando-Navas, D., Aponte-Mayor, G. y Betancourt-Buitrago, L.A. (2014). Metodología para la revisión bibliográfica y la gestión de información de temas científicos, a través de su estructuración y sistematización. Dyna, 81 (184). DOI: http://dx.doi.org/10.15446/dyna.v81n184.37066. 
Ámbitos de aplicación del Coaching educativo: una revisión bibliográfica del periodo 2013-17

Eva Ramos Loredo, Beatriz Sierra-Arizmendiarrieta Y Cristina Roces Montero

Hernández-Aristu, J. y Hernández-Van-Waes, O. (2016). La tutoría en el sistema educativo y el desarrollo de habilidades sociales profesionales (coaching educativo). Aportaciones de la Pedagogía Social y el Trabajo Social. Trabajo Social Global. Revista de Investigaciones en Intervención social, 6 (11), pp. 54-77. http://revistaseug.ugr.es/ index.php/tsg/article/view/4758/pdf

Hoffman, J.V. y Duffy, G.G. (2016). Does Thoughtfully Adaptive Teaching Actually Exist? A Challenge to Teacher Educators. Theory into practice, 55 (3), pp. 172-179. https:// doi.org/10.1080/00405841.2016.1173999

Jiménez, R. (2012). La investigación sobre coaching en Formación del Profesorado: una revisión de estudios que impactan en la conciencia sobre la práctica docente. Profesorado: Revista de currículum y formación del Profesorado, 16 (1), pp. 238-252.

Karpińska-Musiał, B. y Dziedziczak-Foltyn, A. (2014). At students' service: tutoring and coaching as innovative methods of academic education in Poland. En $6^{\text {th }}$ International Conference on Education and New Learning Technologies, pp. 6057-6064. BarceIona, España: IATED. http://www.projektiq.ug.edu.pl/wp-content/uploads/2014/03/ At-students-service-BKMusia\%C5\%82-ADFoltyn.pdf

Kraft, M.A. y Gilmour, A.F. (2016). Can principals promote teacher development as evaluators? A case study of Principals' views and experiences. Educational Administration Quarterly, 52 (5), pp. 711-753. https://doi.org/10.1177/0013161X16653445

Kunz, G. M., White, A. S., Smith, M. H. y Nugent, G. (2014). Coaching Science Inquiry: Validating a Strengths-Based Approach to Coaching. $R^{2}$ Ed Working Paper, 9. http:// r2ed.unl.edu/resources_workingpapers.shtml

Maestre, M.M., Nail, O. y Rodríguez-Hidalgo, A.J. (2017). Desarrollo de competencias TIC y para la educación inclusiva en la formación inicial práctica del profesorado. Bordón, 69 (3), pp. 1-16. https://recyt.fecyt.es/index.php/BORDON/article/view/Bordon.2017.51110/35761

Maldonado, M., Sánchez, T. y Mendoza, G. (2014). Coaching, equipos de trabajo y aprendizaje en organizaciones universitarias. Educare, 18 (2), pp. 92-110.

Matsumura, L. C., Garnier, H. E. y Spybrook, J. (2012). The effect of content-focused coaching on the quality of classroom text discussions. Journal of Teacher Education, 63 (3), pp. 214-228. DOI: 10.1177/0022487111434985. https://doi. org/10.1177/0022487111434985

Mayer, A., Woulfin, S. y Warhol, L. (2015). Moving the center of expertise: Applying a communities of practice framework to understand coaching in urban school reform. Journal of Educational Change, 16 (1), pp. 101-123. DOI: 10.1007/s10833-0149236-y. https://www.csustan.edu/sites/default/files/u2061/moving_the_center_mayer_woulfin_warhol.pdf

Perryman, J., Ball, S.J., Braun, A. y Maguire, M. (2017). Translating policy: governmentality and the reflective teacher. Journal of Education Policy, 32, (6), pp. 745-756. https:// doi.org/10.1080/02680939.2017.1309072

Piper, B. y Zuilkowski, S.S. (2015). Teacher coaching in Kenya: Examining instructional support in public and non formal schools. Teacher and teaching education, 47, pp. 173-183. https://doi.org/10.1016/j.tate.2015.01.001

Purwa Udiutoma, S. T., Srinovita, Y. y Si, S. (2015). The Effect of Coaching and Mentoring 
Ámbitos de aplicación del Coaching educativo: una revisión bibliográfica del periodo

Eva Ramos Loredo, Beatriz Sierra-Arizmendiarrieta Y Cristina Roces Montero

Programs to Improve Students Competencies: Case Study of Beastudi Etos Scholarship. Universal Journal of Educational Research, 3 (3), pp. 163-169. DOI: 10.13189/ ujer.2015.030302. https://files.eric.ed.gov/fulltext/EJ1056088.pdf

Reinke, W. M., Stormont, M., Webster-Stratton, C., Newcomer, L. L., y Herman, K. C. (2012). The incredible years teacher classroom management program: Using coaching to support generalization to real-world classroom settings. Psychology in the Schools, 49 (5), pp. 416-428. DOI: 10.1002/pits.21608. http://www.incredibleyears. com/wp-content/uploads/incredible-years-teacher-training-using-coaching_2012.pdf

Rodríguez-Hidalgo, A.J., Calmaestra Villén, J. y Maestre Espejo, M. (2015). Desarrollo de competencias en el practicum de maestros: ABP y Coaching multidimensional. Profesorado: Revista de currículum y formación del profesorado, 19 (1), pp. 472-493.

Rodríguez Pascual, L. y Martínez Rosillo, V. M. (2015). Efectividad del coaching grupal sobre el desarrollo de la autorregulación del aprendizaje en estudiantes de ingeniería. Cuadernos de Investigación Educativa, 6 (1), pp. 71-88. DOI: http://dx.doi. org/10.18861/cied.2015.6.1.8.

Rosa, G., Riberas, G., Navarro-Segura, L. y Vilar, J. (2015). El Coaching como Herramienta de Trabajo de la competencia Emocional en la Formación de Estudiantes de Educación Social y Trabajo Social de la Universidad Ramón Llul, España. Formación universitaria, 8 (5), (pp. 77-90). DOI: 10.4067/S0718-50062015000500009 https:// scielo.conicyt.cl/scielo.php?script=sci_arttext\&pid=S0718-50062015000500009

Sánchez Mirón, B. y Boronat Mundina, J. (2014). Coaching educativo: Modelos para el desarrollo de competencias intra e interpersonales. Educación XX1, 17 (1), pp. 221242. DOI: 10.5944/educxx1.17.1.107

Sánchez-Teruel, D. (2013). El coaching pedagógico dentro del sistema educativo: innovando procesos. Revista Intercontinental de Psicología y Educación, 15 (2), pp. 171191. http://www.redalyc.org/pdf/802/80228344010.pdf

Sandoval Lentisco, C. y López Martínez, O. (2017). Educacion, psicologia y coaching: un entramado positivo. Educatio Siglo XXI, 35 (1), pp. 145-164. http://dx.doi. org/10.6018/j/286261

Salavert, R. (2015). Coaching: An Apprenticeship Approach for the 21st Century. International Journal of Educational Leadership and Management, 3(1), pp. 4-24. doi: 10.4471/ijelm.2015.02

Slot, P. L., Leseman, P.P.M., Verhagen, J. y Mulder, H. (2015). Quarterly Associations between structural quality aspects and process quality in Dutch early childhood education and care settings. Early Childhood Research Quarterly, 33, pp.64-76.

Twigg, D., Pendergast, D. L., Fluckiger, B., Garvis, S., Johnson, G. y Robertson, J. (2013). Coaching for Early Childhood Educators: An insight into the effectiveness of an initiative. International Research in Early Childhood Education, 4 (1), pp. 73-90. https:// research-repository.griffith.edu.au/handle/10072/55703

Van der Weijden, I., Teelken, C., de Boer, M. y Drost, M. (2016). Career satisfaction of postdoctoral researchers in relation to their expectations for the future. Higher Education, 72 (1), pp. 25-40. https://doi.org/10.1007/s10734-015-9936-0

Van Lankveld, T., Schoonenboom, J., Kusurkar, R.A., Volman, M., Beishuizen, J. y Croiset, J. (2017). Integrating the teaching role into one's identity: a qualitative study of begin- 
Ámbitos de aplicación del Coaching educativo: una revisión bibliográfica del periodo 2013-17

Eva Ramos Loredo, Beatriz Sierra-Arizmendiarrieta Y Cristina Roces Montero

ning undergraduate medical teachers. Advances in Health Sciences Education, 22(3), pp . 601-622. doi: 10.1007/s10459-016-9694-5 https://www.ncbi.nlm.nih.gov/pmc/ articles/PMC5498609/

Vernon-Feagans, L., Kainz, K., Hedrick, A., Ginsberg, M. y Amendum, S. (2013). Live webcam coaching to help early elementary classroom teachers provide effective literacy instruction for struggling readers: The Targeted Reading Intervention. Journal of Educational Psychology, 105 (4), pp. 1175-1187. DOl: 10.1037/a0032143.

Wang, Q. y Millward, I. (2014). Developing a unified psychological model of coaching and mentoring in supporting the learning and development of adolescents. International Journal of Evidence Based Coaching and Mentoring, 12 (2), pp. 91-108. http:// ijebcm.brookes.ac.uk/documents/vol12issue2-paper-07.pdf

Whitmore, J. (1992). Coaching for Performance. Growing human potencial and purpose. The principles and practice of coaching and leadership. London: Nicholas Brealey Publishing.

Yee, L. W., (2016). Peer Coaching for Improvement of Teaching and Learning. Journal of Interdisciplinary Research in Education, 6 (1), pp. 64-70. https://university2.taylors. edu.my/jire/downloads/vol6_05.pdf 